\title{
Hypoxia induces therapeutic resistance and enhances subsequent reformulated tumorigenesis and metastasis
}

\author{
Lawrence M. Agius* \\ Department of Pathology, Mater Dei Hospital, Tal-Qroqq, University of Malta Medical School, Msida, Malta Europe
}

\begin{abstract}
Dynamics of hypoxic environment generation within tumors are major pathogenic factors in the emergence of radio- and chemo-resistance of multiple diverse types of neoplasia. It is significant to consider various approach modalities in the treatment of tumors that are often significantly enhanced by hypoxia in further generating malignant transformation and progression. Focusing modalities implicate also repair mechanisms involved in correcting mutations such as insertions/deletions, translocations and base-damage. Such DNA repair pathways have direct impact on the outcome of radiotherapy and also chemotherapy that induce DNA lesions in therapeutically enhanced tumor cell death or apoptosis.
\end{abstract}

\section{Introduction}

Hypoxia constitutes a self-amplifying evolutionary spectrum in the biologic progression span of many or most solid human tumors. The cellular iron importer, divalent metal transporter 1 is strongly expressed in colorectal carcinomas through hypoxia-inducible factor 2alphadependent transcription [1]. Targeting hypoxia is believed to be the best validated strategy in cancer treatment [2]. Incremental dimensions include the acquisition of contextual synthetic lethality in specific modes of combined genetic lesions within a given cellular genome. It is with respect to hypoxia-induced resistance to radiotherapy and also chemotherapy that there seems to evolve a series of suppressive effects on DNA-repair pathways within such extensive regions of severe hypoxic regions within tumors. Hypoxia, tumor acidification, cancer stem cells, exosomes and also protein transporters, apoptosis dysregulation and angiogenesis in a tumor micro-environment may contribute to therapy resistance [3]. Down-regulation of apoptosisinducing factor by hypoxia controls epithelial-mesenchymal transition of cancers, with oxidative inactivation of PTEN [Phosphatase and tensin homolog on chromosome ten] and subsequent activation of Akt kinase and of WNT/beta-catenin signaling [4]. Defined indices of progression of hypoxic solid tumors relate particularly to the actual malignant transformation and progression that include metastasisgeneration in various systemic organs such as the lungs. In invasive bladder cancer, cell-surface Sialyl-Tn antigen over-expression enhances migration and invasion in a HIF-1alpha-dependent manner [5]. The further conformational dimensions of hypoxia include the combined processes of acute and also chronic hypoxia that further involve spectrum creation of suppression of checkpoint progression. Inhibition of the CXCL 12/CXCR4 signaling axis in glioblastoma induces apoptosis and blocks cell cycle progression and proliferation and also inhibits hypoxic-induction of HIF-1alpha [6].

\section{Acute with chronic hypoxia}

Combined acute and chronic hypoxia affects multiple regions of a solid human tumor and this is believed to be the main cause of radio-resistance in tumor therapy. In vivo methods to measure oxygen in tissue have important clinical potential [7]. Also, adverse tumor microenvironments such as hypoxia and extracellular acidosis affect the expression of inflammatory mediators and hence immune response within tumors [8]. The incremental emergence of thrombosis of tumor vessels and the bizarre and haphazard organization of the tumor vasculature compound such hypoxia in further promoting radio-resistance of the tumor cells. Improvements by model-based methods for the estimation of tumor oxygenation are in agreement with experimental data, involving computational modeling and pharmacokinetic modeling [9]. Mechanistic approaches utilizing such measures as hyperbaric oxygenation and the administration of erythropoietin fail to effectively sensitize many regions of tumor hypoxia and hence constitute a truly independent parameter in therapeutic resistance. Pathogenesis of hypoxia is multifactorial in tumors and spatial distribution often changes over time [10]. Intensity modulatory radiotherapy has been employed to deal with relatively resistant hypoxic tumor regions, and impaired DNA repair pathways in multiple foci of the lesion may promote such effects.

\section{Repair inhibition}

Mismatch repair inclusively modulates the response of neoplastic cells to chemotherapeutic agents. Therapeutic approaches involving cellular uptake of such chemotherapeutic agents may be offset by the protonation of cytotoxic agents, with failure of uptake of the chemicals from the tumor extracellular environment and its decreased $\mathrm{pH}$. Cholesterol induces membrane resistance at the tissue level, contributing to the Warburg effect in tumors and reducing oxygen permeability [11]. Gradients of operative alkylation and the hypoxia-

Correspondence to: Lawrence M. Agius, Department of Pathology, Mater Dei Hospital, Tal-Qroqq, University of Malta Medical School, Msida, Malta Europe, Tel: 35621451752; E-mail: lawrence.agius@um.edu.mt

Key words: hypoxia, tumorigenesis, metastasis, DNA

Received: September 19, 2016; Accepted: December 19, 2016; Published: December 23, 2016 
targeting of tumor cells have been modulated in an attempt to sensitize tumor cells to therapeutic agents in a manner that is only relatively effective. HIF signaling serves as a major adaptive mechanism in hypoxic tumors, promoting direct growth-enhancing cytokine production, angiogenesis and reactive oxygen species production [12].

Biophysical vicinity of oxygen free radicals to cellular DNA is an important consideration in tumor therapeutics and one that may be utilized in a loco-regional fashion, when administering radiotherapy. Esterified 3-oxoglutarate targeting of HIF-1alpha in combination with use of existing cytotoxic agents can serve as potent antitumor chemotherapies [13]. Ionizing radiation can potentially add bulky adducts and thus distort the bi-hilar structure of DNA. "Fixing" of lesions to the cellular DNA molecule can be achieved with the use of fractionated radiotherapy in inducing double-strand breaks, with also an attempt at preserving an important therapeutic ratio with regard to normal surrounding tissues. In this manner, one may achieve more effective targeting of the tumor cells.

\section{Hypoxia-inducible factor-1alpha}

Hypoxia-inducible factor-1alpha controls the cell responses to hypoxia. Such mechanisms allow for the development of activated promoters. Hypoxia is considered a poor prognostic in tumor progression and metastasis in a manner that permits unrepaired tumor cells that clonally divide and the subsequent emergence of more aggressive groups of neoplastic cells. Also, acidosis is an important regulator of 2-hydroxyglutarate with implications for HIF signaling in terms of epigenetic control [14]. The essential association of hypoxia with mutator phenotype includes such phenomena as the involvement of mutant p53 tumor cells that are resistant to apoptosis-induced cell death. Altered gene expression may be coupled to delayed cell cycling checkpoints in regions of tumor hypoxia and may thus transform cells with regard to maturation and slow rates of proliferation.

\section{Hypoxia-responsive genes}

Profiles of hypoxia-responsive gene expression prove a derivative dysfunctionality in terms of the emergence of a tumor evolutionary course. There is considerable heterogeneity of fractional cerebral blood volume, oxygen saturation and blood oxygenation level within multiple tumor compartments including core, rim and peritumor regions; also, the flow/metabolism interactions prove highly complex [15]. A cell-cycle arrest induces displacement of Myc and inhibitory p21 waf; the latter is a centrally operative cyclin-dependent kinase inhibitor controlling the G1 checkpoint.

Repair and checkpoint proteins such as BRCA1, RAD51 and retinoblastoma may thus come to operate independently of hypoxia-inducible factor lalpha and thus there emerges new sets of gene expression; such phenomenon may constitute empowered transforming potentiality in hypoxic tumor cells.

HIF-1alpha may inhibit translational activity either through the unfolded protein response as a hypoxic stress phenomenon, or through mRNA translational inhibition of initiation. eIF4F dysfunction leads to a failed transcript recruitment step in mRNA translation.

\section{Transcription and translation in DNA repair}

Transcriptional repression exerted by hypoxia is a realized dimension in the ongoing acquisition of resistance to radiotherapy and ionizing radiation. It is further to the extensive development of aberrant angiogenesis within solid tumors that the comparative profiles of DNA repair pathways are themselves defining parameters of carcinogenesis per se. It is in such terms that the whole DNA polymerase beta and polymerase delta pathways include failure of DNA repair pathways in hypoxia.

Inclusion phenomena involve the cell-cycle checkpoint suppression of the individual phase of the life-span of the proliferating individual tumor cell. Down-regulation of HIF pathway by Lactobacillus rhamnosus and of HSP90 expression may have anti-cancer effects [16]. The whole panorama of dimensional reduction of such inclusive dynamics allow for permissive genomic instability towards the subsequent emergence and establishment of the mutator phenotype of that individual tumor cell. FAT Atypical Cadherin1-HIF-1alpha axis may control the invasiveness of glioblastoma with also suppression of tumor inflammation [17], The angiogenic vessels of a solid tumor comprise a particular susceptibility to diffusion failure of the oxygen supply to the neoplastic cells. Distances beyond the 150u limit from the abnormal leaky vessels would specify a further promotional series of events that couple to intra-vascular supply that is implicit in long transit times within the angiogenic vessels of a given tumor.

\section{Cyclic hypoxia}

Cyclic oxygenation may constitute a series of mechanisms as utilized in fractionated radiotherapy, with the generation of oxygen free radicals and double strand breaks in the cellular DNA. The deposition of extracellular matrix in pancreatic ductal adenocarcinoma may involve the production of cytotoxic levels of reactive oxygen species [18]. Homologous recombination and nucleotide excision endjoining may operate in the global genome in a transcriptional-specific manner in producing repair of gaps in the DNA. Genomic instability in mutator phenotypes promotes carcinogenesis per se, in a manner that further facilitates the emergence of both tumor cell transformation and also towards the formation of increasingly aberrant angiogenic vessels. Higher tumor hypoxia levels have predictive value in head and neck cancer with lower overall survival and impaired loco-regional control [19].

\section{Chemotherapy resistance}

Multiple operative factors are implicated in tumor resistance to chemotherapy in hypoxia, and molecular, cellular and extracellular mechanisms are involved. Metabolic reprogramming and tumor growth models indicate the necessity for repression of pyruvate dehydrogenease under hypoxic conditions [20]. Poor agent delivery and the involvement of HIF-1alpha in inducing regulated chemoresistance may facilitate regulation of many genes. (18F)FMISO-PET provides an opportunity to predict response to HER2+ breast cancer before changes in tumor size develop and may identify decreased hypoxic regions important in guiding subsequent therapy [21].

HIF-1alpha is particularly implicated in inducing a shift of proapoptotic factors to anti-apoptosis, thus contributing to tumorigenesis and tumor progression. Inhibition of HIF-1alpha provides immune adjuvant activity, thus improving the efficacy of tumor antigen-based dendritic cell vaccination [22]. Chemotherapeutic agents in general induce potential mutagenesis of genes that may not be easily reparable within a context of carcinogenesis. The presence of two or more genetic lesions in a given cellular genome may enhance further genetic instability in terms of contextual synthetic lethality.

The core tumor region involves interactivity of hypoxia-surviving cells with an inflammatory microenvironment and newly recruited 
stromal cells and tumor-associated macrophages; this constitutes an aggressiveness niche prone to metastatic spread [23].

\section{Novel formulated approaches}

A schedule of accelerated radiotherapy constituted by antiproliferative effect, carbogen that counteracts diffusion-enhanced hypoxia, and nicotinamide to enhance vasoreactivity, has been effectively utilized in head and neck tumors and advanced bladder carcinoma. Heterogeneity of hypoxic cell response is also related to the status of biomarkers of hypoxia that respond to other types of tumor cell conditioning. Intensity-modulated radiotherapy may be employed in conjunction with positron emission tomography imaging and constitutes another approach to radiation of hypoxic regions of tumors. Hypoxia-activated pro-drugs include dinitrobenzamide mustards when activated generate oxygen free radical metabolites appear superior to other bioreductive drugs such as tirapazamine; activation can occur at lower oxygen tensions with greater specificity, and this is coupled to greater diffusion of the tumor interstitium.

\section{Concluding remarks}

Various measures to overcome hypoxia-induced radio-resistance and chemo-resistance have proved relatively ineffective in regions of tumor hypoxia. A central problem is the operative involvement of multiple pathogenic factors implicated in decreasing oxygen tension within different neoplastic types. HIF-1alpha also appears to be activated by factors other than hypoxia. Glioma hypoxia and acidosis functionally cooperate to induce HIF transcription factors and preservation of cancer stem-like cells [24]. Also, it is important to discriminate between acute and chronic hypoxia in a manner inherently linked to pathogenic pathways of tumor cell proliferation and progression. One particular aspect implicates hypoxia in the malignant transformation process and also in subsequent tumor progression and metastasis. Loco-regional phenomena of hypoxiainduction are central issues and would be suggestive of important roles in strictly focusing distinct regions of hypoxia in tumors less than 10 $\mathrm{mm} \mathrm{Hg}$ oxygen tension.

\section{References}

1. Xue X, Ramakrishnan SK, Weisz K, Triner D, Xie L, et al. (2016) Iron Uptake via DMT1 Integrates Cell Cycle with JAK-STAT3 Signaling to Promote Colorectal Tumorigenesis. Cell Metab 24: 447-461. [Crossref]

2. Kang L, Fan B, Sun P, Huang W, Jin M, et al. (2016) An effective tumor-targeting strategy utilizing hypoxia-sensitive siRNA delivery system for improved anti-tumor outcome. Acta Biomater 44: 341-354. [Crossref]

3. Fraczek N, Bronisz I, Pietryka M, Kepinska D, Strzala P, et al. (2016) An outline of main factors of drug resistance influencing cancer therapy. $J$ Chemother 28: 457-464. [Crossref]

4. Xiong Z, Guo M, Yu Y, Zhang FF, Ge MK, et al. (2016) Down regulation of AIF by HIF-1 contributes to hypoxia-induced epithelial-mesenchymal transition of colon cancer. Carcinogenesis 37(11): 1079-1088.

5. Peixoto A, Fernandes E, Gaiteiro C, Lima L, Azevedo R, et al. (2016) Hypoxia enhances the malignant nature of blader cancer cells and concomitantly antagonizes protein O-glycosylation extension. Oncotarget 7(39):63138-63157. [Crossref]

6. Calinescu AA, Yadav VN, Carballo E, Kadiyala P, Tran D, et al. (2016) Survival and proliferation of neural progenitor derived glioblastomas under hypoxic stress is controlled by a CXCL 12/CXCR4 autocrine positive feedback mechanism. Clin Cancer Res, Epub ahead of print.

7. Flood AB, Satinsky VA, Swartz HM (2016) Comparing the Effectiveness of Methods to Measure Oxygen in Tissues for Prognosis and Treatment of Cancer. Adv Exp Med Biol 923: 113-120. [Crossref]

8. Riemann A, Ihling A, Reime S, Gekle M, Thews O (2016) Impact of the Tumor
Microenvironment on the Expression of Inflammatory Mediators in Cancer Cells. $A d v$ Exp Med Biol 923: 105-111. [Crossref]

9. Shi K, Ziegler SI, Vaupel P (2016) Molecular Imaging of Tumor Hypoxia: Existing Problems and Their Potential Model-Based Solutions. Adv Exp Med Biol 923: 87-93. [Crossref]

10. Vaupel P, Mayer A (2016) Tumor hypoxia: causative mechanisms, microregional heterogeneities, and the role of tissue-based hypoxia markers. Adv Exp Med Biol 923: $77-86$.

11. Shea R, Smith C, Pias SC (2016) Magnification of cholesterol-induced membrane resistance on the tissue level: implications for hypoxia. Adv Exp Med Biol 923: 43-50.

12. Triner D, Shah YM (2016) Hypoxia-inducible factors: a central link between inflammation and cancer. J Clin Invest 126: 3689-3698. [Crossref]

13. Koivunen P, Fell SM, Lu W, Rabinowitz JD, Kung AL, et al. (2016) The 2-oxoglutarate analog 3-oxoglutarate decreases normoxic hypoxia-inducible factor-1alpha in cancer cells, induces cell death, and reduces tumor xenograft growth. Hypoxia (Auckl) 4: 1527.

14. Nadtochiy SM, Schafer X, Fu D, Nehrke K, Munger J, et al. (2016) Acidic pH Is a Metabolic Switch for 2-Hydroxyglutarate Generation and Signaling. J Biol Chem 291 20188-20197. [Crossref]

15. Golias T, Papandreou I, Sun R, Kumar B, Brown NV, et al. (2016) Hypoxic repression of pyruvate dehydrogenase activity is necessary for metabolic reprogramming and growth of model tumors. Sci Rep 6: 31146 .

16. Esfandiary A, Taherian-Esfahani Z, Abedin-Do A, Mirfakhraie R, Shirzad M, et al (2016) Lactobacilli modulate hypoxia-inducible factor (HIF)-1 regulatory pathway in triple negative breast cancer cell line. Cell J 18(2): 237-244.

17. Madan E, Dikshit B, Gowda SH, Srivastava C, Sarkar C, et al. (2016) FAT1 is a novel upstream regulator of HIF 1 alpha and invasion of high grade glioma. Int J Cancer 139 2570-2582. [Crossref]

18. Topalovski M, Hagopian M, Wang M, Brekken RA (2016) Hypoxia and Transforming Growth Factor beta Cooperate to Induce Fibulin-5 Expression in Pancreatic Cancer. $J$ Biol Chem 291: 22244-22252. [Crossref]

19. Clavo B, Robaina F, Fiuza D, Ruiz A, Lioret M, et al. (2016) Predictive value of hypoxia in advanced head and neck cancer after treatment with hyperfractionated radio-chemotherapy and hypoxia modification. Clin Transl Oncol, Epub ahead of print.

20. Chakhoyan A, Corroyer-Dulmont A, Leblond MM, Gerault A, et al. (2016) Carbogeninduced increases in tumor oxygenation depend on the vascular status of the tumor: a multiparametric MRI study in two rat glioblastoma models. J Cereb Blood Flow Metab.

21. Sorace AG, Syed AK, Barnes SL, Quaries CC, Sanchez V, et al. (2016) Quantitative [18F]FMISO PET imaging shows reduction of hypoxia following trastuzumab in a murine model of HER2 + breast cancer. Mol Imaging Biol.

22. Kheshtchin N, Arab S, Ajami M, Mirzaei R, Ashourpour M, et al. (2016) Inhibition of HIF-1 1 I enhances anti-tumor effects of dendritic cell-based vaccination in a mouse model of breast cancer. Cancer Immunol Immunother 65: 1159-1167. [Crossref]

23. ElShamy WM, Sinha A, Said N (2016) Aggressiveness Niche: Can It Be the Foster Ground for Cancer Metastasis Precursors? Stem Cells Int 2016: 4829106. [Crossref]

24. Filatova A, Seidel S, Bogurcu N, Graf S, Garvalov BK, et al. (2016) Acidosis Acts through HSP90 in a PHD/VHL-Independent Manner to Promote HIF Function and Stem Cell Maintenance in Glioma. Cancer Res 76: 5845-5856. [Crossref]

Copyright: (C2016 Agius LM. This is an open-access article distributed under the terms of the Creative Commons Attribution License, which permits unrestricted use, distribution, and reproduction in any medium, provided the original author and source are credited. 